\title{
Declining trends in early warning indicators for HIV drug resistance in Cameroon from 2008-2010: lessons and challenges for low-resource settings
}

Joseph Fokam ${ }^{1,2^{*}}$, Serge C Billong ${ }^{2,3^{*}}$, Anne C ZK Bissek ${ }^{2,4}$, Etienne Kembou ${ }^{5}$, Pascal Milenge ${ }^{5}$, Ibile Abessouguie ${ }^{1}$, Armand S Nkwescheu ${ }^{2,6}$, Zephirin Tsomo ${ }^{7}$, Avelin F Aghokeng ${ }^{8}$, Grace D Ngute ${ }^{3,9}$, Peter M Ndumbe ${ }^{1,2,10}$, Vittorio Colizzi ${ }^{1,11}$ and Jean BN Elat ${ }^{2}$

\begin{abstract}
Background: Rapid scale-up of antiretroviral therapy (ART) and limited access to genotyping assays in low-resource settings (LRS) are inevitably accompanied by an increasing risk of HIV drug resistance (HIVDR). The current study aims to evaluate early warning indicators (EWI) as an efficient strategy to limit the development and spread of preventable HIVDR in these settings, in order to sustain the performance of national antiretroviral therapy (ART) rollout programmes.

Methods: Surveys were conducted in 2008, 2009 and 2010 within 10 Cameroonian ART clinics, based on five HIVDR EWIs: (1) Good prescribing practices; (2) Patient lost to follow-up; (3) Patient retention on first line ART; (4) On-time drug pick-up; (5) Continuous drug supply. Analysis was performed as per the World Health Organisation (WHO) protocol.

Results: An overall decreasing performance of the national ART programme was observed from 2008 to 2010: $\mathrm{EWI}_{1}$ (100\% to $70 \%) ; \mathrm{EWI}_{2}$ (40\% to $20 \%$ ); $\mathrm{EWI}_{3}$ (70\% to $0 \%$ ); $\mathrm{EWI}_{4}$ (0\% throughout); $\mathrm{EWI}_{5}$ (90\% to $40 \%$ ). Thus, prescribing practices $\left(\left.E W\right|_{1}\right)$ were in conformity with national guidelines, while patient adherence $\left(\mathrm{EWI}_{2}, \mathrm{EWI}_{3}\right.$, and $\left.\mathrm{EWI}_{4}\right)$ and drug supply $\left(\mathrm{EWI}_{5}\right)$ were lower overtime; with a heavy workload (median ratio $\approx 1 / 64$ staff/patients) and community disengagement observed all over the study sites.

Conclusions: In order to limit risks of HIVDR emergence in poor settings like Cameroon, continuous drug supply, community empowerment to support adherence, and probably a reduction in workload by task shifting, are the potential urgent measures to be undertaken. Such evidence-based interventions, rapidly generated and less costly, would be relevant in limiting the spread of preventable HIVDR and in sustaining the performance of ART programmes in LRS.
\end{abstract}

Keywords: Early warning indicator, HIV drug resistance, Surveillance and prevention, Cameroon

\footnotetext{
*Correspondence: fokamjoseph2002@yahoo.fr; sergebillong@yahoo.fr

${ }^{1}$ Chantal BIYA International Reference Centre (CIRCB) for research on HIV/

AIDS prevention and management, Yaounde, Cameroon

${ }^{2}$ Faculty of Medicine and Biomedical Sciences (FMBS) of the University of

Yaounde 1, Yaounde, Cameroon

Full list of author information is available at the end of the article
} 


\section{Background}

Low- and middle-income countries had just over 8 million people receiving highly active antiretroviral therapy (HAART) by end of 2011, representing 54\% [50-60\%] coverage of eligible patients based on World Health Organisation (WHO) guidelines $(\mathrm{CD} 4 \leq 350$ cells/ $\mu \mathrm{l})$ [1]. As compared to 2010 (<6 million) and 2003 (400,000), this coverage has been greatly favored by the rapid scale-up of antiretroviral therapy (ART), with sub-Saharan Africa being the main beneficiary (6.2 million people were receiving antiretroviral therapy in 2011, up from just 100,000 in 2003) $[1,2]$. In Cameroon, these efforts led to reductions in AIDS-associated morbidity and mortality, and a relative decrease in HIV prevalence (from $5.5 \%$ in 2004 to $4.3 \%$ in 2011), with close to $44.5 \%$ eligible patients on ART [3,4]. Furthermore, the number of ART clinics and of treated patients has increased overtime: 5 clinics for 116 (1.3\%) eligible patients in 2002, to 145 clinics for 89,455 (36\%) eligible patients in 2010 [4-6]. Since scale-up of ART is known to be associated with a high risk of HIV drug resistance (HIVDR), strategies to combat HIVDR are of public health priority in Cameroon [6-8]. Most importantly, with limited access to reference laboratory equipment (CD4 count, HIV viral load, HIVDR testing) and the use of drugs with low genetic barrier for resistance, HIVDR population-based surveillance and prevention are recommended to ensure long term efficacy of treatment guidelines [8-11]. As response to this crucial need, a national HIVDR working group (HIVDRWG) was created based on the WHO global HIVDR prevention and assessment strategy which includes the laboratory-based surveys of transmitted and acquired HIVDR, and monitoring of HIVDR early warning indicators (EWIs); with the guidelines being recently revised and updated in 2012 [12-17]. Surveys in Namibia, Malawi, South-Africa, and other African settings [18-24]; in Central American and Caribbean countries, as well as in Asia and Oceania (Papua New Guinea) [23-31], also supported the use of EWIs to evaluate the risk of ART failure and HIVDR emergence. Such evaluations use existing clinic- and pharmacy-based data on ART prescribing at the ART clinic $[12,15,16]$. Six strongly recommended and two optional EWIs are proposed by the WHO, among which $\geq 4$ feasible EWIs should be chosen for an effective ART programme evaluation [15]. In this prospect, we aimed to evaluate the levels and trends of five WHOstrongly recommended EWIs, in order to identify potential strengths and weaknesses (i.e. gaps in service delivery that might inform policy changes to improve performance) of the national ART program and to target appropriate interventions that can optimize care and potentially minimize the risk of emerging resistant patterns that could compromise the commonly used antiretrovirals in the country. Of note, results presented in this study are based on WHO HIV drug resistance early warning indicator guidance [15], and not upon the updated 2012 guidance as presented in the latest WHO HIV drug resistance early warning indicator meeting report [16].

\section{Methods}

\section{Study design and population}

Using a retrospective review of clinical data from ART sites in Cameroon, five early warning indicators were assessed in 2008, 2009 and 2010 within 10 Cameroonian ART clinics. These ART clinics were chosen based on criteria among which: the number of years of experience on ART management ( $\geq 3$ years), the frequency of new enrollments on ART per quarter (i.e. $\geq 30$ newly enrolled patients on ART every 3 months), the geographical locations of the ART clinic over the national territory, and finally on convenience amongst sites meeting these criteria. At the beginning of the study in 2008, 133 ART clinics \{24 approved treatment centers [ATCs] and 109 HIV management units: [HMUs]\} existed nationwide, among which 89 were already operational since 2005 . Thus, based on the above mentioned eligibility criteria ( $\geq 3$ years experience), our study coverage represented a proportion of $\approx 11 \%(10 / 89)$ of the entire national ART clinics.

The five EWIs were selected and defined according to the WHO criteria $[12,15]$ :

- EWI 1 : "Good ARV prescribing practices" (Percentage of patients initiated on an appropriate first line ARV drug regimen). Numerator: Number of individuals initiating ART at the site who are prescribed a standard or otherwise appropriate first-line regimen during the selected time period. These are first line regimens recommended by the country national guidelines, and exclude dual- or mono-therapy. Denominator: Number of individuals starting ART during the selected time period. The acceptable target performance: $100 \%$.

- EWI 2 : "Patient lost to follow-up" (Percentage of patient lost to follow-up after 12 months of enrolment to ART). Numerator: Number of individuals initiating ART in a selected time period that were not seen at the clinic or pharmacy $\geq 90$ days after the date of their last missed appointment or drug pick-up that occurred within their first 12months of ART, and who are not known to have transferred out or died. Denominator: Number of individuals starting ART during a selected time period. The acceptable target performance $\leq 20 \%$.

- EWI $\mathrm{EL}_{3}$ "Patient retention on appropriate first line ART" (Percentage of patient retention on appropriate first line ART after 12 month of treatment). Numerator: Number of individuals 
initiating first-line ART during a selected period of time who are, 12 months from ART start, still on first-line ART (this includes substitutions of one appropriate first-line regimen for another, but not substitutions of dual- or mono-therapy or an inappropriate three-drug regimen). Denominator: Number of individuals starting ART during a selected time period or, in sites where data are available, that number minus the number of individuals starting ART in that time period who transferred out during the 12 months after starting ART. Individuals who died, stopped ART, switched to second-line ART, or were lost to follow-up must be included in the denominator. The acceptable target performance $\geq 70 \%$.

- EWI 4 : "On-time ARV drug pick-up" (Percentage of on-time ARV drug pick-up by the patient).

Numerator: number of individuals who have picked up all their prescribed ARV drugs on time during the selected time period. This EWI uses a sample of all patients on ART, and follows them for two drug pick-ups after baseline pick-up. Denominator: number of individuals classified as "on ARV drugs" during the selected time period. The acceptable target performance $\geq 90 \%$.

- EWI: "Drug supply continuity" (Percentage of ARV drug supply continuity at the site pharmacy). Numerator: Number of months or quarters in the year in which there were no ARV drug stock outages for any ARVs in any of the standard ART regimens supplied by the site or the pharmacy at which the site's patients pick up ARV drugs. Denominator: 12 months. The acceptable target performance: $100 \%$.

Sample size for each ART site was calculated based on the WHO guidance. In detail, for the first year (2008), considered as the ever first or pilot phase for such study in the country, a sample size of thirty (30) patients per site was required. Patient sampling for the two-other yearly evaluations (2009-2010) was then based on the total number of new enrollments at ART during the study year, with respect to the following WHOrequirements shown in Table 1 . This sampling method derived from the following formula: $\mathrm{N}=\left(\mathrm{Z}^{2} \times \mathrm{P} \times \mathrm{Q}\right) /$ $\mathrm{d}^{2}$; with $\mathrm{N}$ being the minimum sample size, $\mathrm{Z}$ the confidence interval at $95 \%(\mathrm{Z}=1.96), \mathrm{P}$ the expected percentage of retention on ART, Q as 1- P, and D the precision at a degree of accuracy $(\mathrm{d}=7 \%)[12,15]$.

\section{Data collection, validation and analysis}

Description of each ART site was carried out as per WHO suggested "site profile", which includes the category of the site (ATC or HMU), the geographical
Table 1 WHO-sampling method $[12,15]$

\begin{tabular}{ll}
\hline $\begin{array}{l}\text { Number of newly enrolled patients } \\
\text { on ART per year }\end{array}$ & Required sample size \\
\hline $1-75$ & Recruit all \\
$76-110$ & 75 \\
$111-199$ & 100 \\
$200-250$ & 110 \\
$251-299$ & 120 \\
$300-350$ & 130 \\
$351-400$ & 135 \\
$401-450$ & 140 \\
$451-550$ & 145 \\
$551-700$ & 155 \\
$701-850$ & 160 \\
$851-1600$ & 175 \\
$1601-2150$ & 175 \\
$2151-4340$ & 200 \\
$4341-5670$ & 210 \\
$5671-10,000$ & 215 \\
$>10,000$ & WHO advice required \\
\hline$W H 0: W 01 d-40 a t h 0$ &
\end{tabular}

WHO: World Health Organization.

localization, years of experience in ART management, the number of patients on ART, number and category of trained staff, availability of treatment guidelines, and availability of first/second line ARV drugs.

Data were collected using WHO data abstraction tools (standardized sheets) among patients newly enrolled on ART and followed-up zone year. Briefly, the first three EWIs were obtained by abstraction of cumulative cohort data from ART registers, while the two other EWIs were abstracted from the pharmacy register. In order to ensure reliability in the quality of data, data verification/ validation was performed using $20 \%$ of data from the medical records. Validation was a two levels process: firstly on-site (by source verification in ART and pharmacy registers, against the WHO standardized data collection sheets), and then secondly at the central level (by verification between the standardized collection sheet against the WHO-electronic routine data quality assessment tool: "RDQA"). The levels of EWIs were obtained each year, and the trends were evaluated through, a chronological analysis, using these 10 ART clinics enrolled continuously throughout the three years (2008, 2009 and 2010), and statistically significance overtime was analyzed for the performance of each EWI using the Fisher exact test, with a p-value $<0.05$ considered as a statistically significant difference. EWI attaining the acceptable target was classified as "Good Performance", while EWI unable to reach the target was classified as "Poor Performance". 


\section{Ethical considerations}

With respect to the Helsinki Declaration for research carried out on humans (including human material or human data), ethical approval was obtained from the National Ethics Committee (Authorization N ${ }^{\circ} 034 / \mathrm{NEC} /$ $\mathrm{SE})$, Yaounde, Cameroon. Confidentiality was ensured during data abstraction by the use of identification codes. Since the study focused on ART site performance rather than individual patient, informed consent from patients was not required. For on-site knowledge transfer and owning of EWI activities to staff, heads of the ART clinics and their respective data managers were preliminarily trained on HIVDR EWIs and on the methodology for data collection, analysis and interpretation.

\section{Results}

\section{Description of the ART clinics}

Data validation was successful for all the 10 ART clinics throughout the three years (2008, 2009 and 2010). These ART clinics were geographically distributed in four different regions of the country, among which four sites from the Centre region (Yaounde General Hospital, National Social Welfare Hospital, Yaounde Central Hospital, and Yaounde Jamot Hospital); three sites from the Littoral region (Douala General Hospital, Laquintinie Hospital Douala, and Nylon District Hospital); two sites from the North-west region (Bamenda Regional Hospital, and Polyclinic Mezam Bamenda); one site from the South-west region (Limbe Regional Hospital).

These ART clinics had a median working experience in the HIV/AIDS therapeutic management of 6.5 years [Interquartile range, IQR: 5-7]. The national ART guidelines were available in all the sites, as well as treatment protocols for first line ARV drugs; second line drug regimens were available solely in ATCs. ARV drugs were provided at a pharmacy within the clinic; and drug stocks were run using designed sheets.

Throughout the study, each of the clinics had more than 30 patients newly enrolled on ART quarterly (i.e. in every three months period). By the end point (i.e. in 2010), a total 27,826 patients were on ART in the entire study clinics, and staffs (physicians, nurses, pharmacists/ clerks, biologists/laboratory technicians, counselors, data managers, community relay agents) were all trained on the routine management of people living with HIV. The staff-patient distribution per site was as follows: Yaounde General Hospital (1,542 patients for 24 staffs; $\approx 64 / 1$ ratio), National Social Welfare Hospital (1,247 patients for 178 staffs; $\approx 6 / 1$ ratio), Yaounde Central Hospital $(6,031$ patients for 43 staffs; $\approx 140 / 1$ ratio $)$, and Yaounde Jamot Hospital (1,610 patients for 33 staffs; $\approx 49 / 1$ ratio), Douala General Hospital (2,049 patients for 34 staffs; $\approx 61 / 1$ ratio), Laquintinie Hospital Douala (3,831 patients, staffs number not provided), Nylon District
Hospital (2,800 for 34 staffs; $\approx 83 / 1$ ratio), Bamenda Regional Hospital (4,032 patients for 31 staffs; $\approx 130 / 1$ ratio), Polyclinic Mezam Bamenda (2,100 patients for 18 staffs; $\approx 117 / 1$ ratio), and Limbe Regional Hospital (2584 patients for 191 staffs; $\approx 14 / 1$ ratio). Thus, this resulted to an overall median staff-patient ratio of $\approx 1 / 64$ [Interquartile range (IQR): 1/49 - 1/117], indicative of a heavy workload; interestingly, the only ART site with $\geq 50 \%$ good performance over the three years survey (H. CNPS: $60 \%$ good performance i.e. $9 / 15$, as shown in Table 1) had the lowest workload (1/6 staff/patient ratio). An overview distribution of patients per staff category was as follows: medical doctor (1:267 patients); nurse (1:74 patients); biologists/laboratory technicians (1:434 patients); pharmacist/pharmacy clerk (1:694 patients); counselor (1:434 patients); community relay agent (1:323 patients); and data manager (1:1387 patients).

\section{Performances of EWIs in 2008, 2009, and 2010}

For $\mathrm{EWI}_{1}$, the overall yearly performance moved from $100 \%(10 / 10)$ in $2008,80 \%(8 / 10)$ in 2009 , to $70 \%(7 / 10)$ in 2010; without any statistically difference overtime $(\mathrm{p}=$ 0.47). Of note, the few sites with suboptimal performances had each a target of $98-99 \%$, which is much closed to the required performance of $100 \%$. No dual- or mono-therapy was reported in any of the studied clinics.

For $\mathrm{EWI}_{2}$, only $40 \%(4 / 10)$ reached the required target $(\leq 20 \%)$ in 2008 against $20 \%(2 / 10)$ in 2009 and $20 \%(2 / 10)$ in 2010; revealing an increasing rate of patients lost to follow-up between the first two years, followed by stable rate; still without any statistically significant difference over time $(\mathrm{p}=0.82)$.

For $\mathrm{EWI}_{3}, 70 \%(7 / 10)$ of sites reached the required performance target $(\geq 70 \%)$ in 2008 , against $10 \%(1 / 10)$ and $0 \%(0 / 10)$ in 2009 and 2010, respectively; describing an overall decreasing performance in terms of patient retention on first line ART, with a statistically significant difference overtime $(\mathrm{p}=0.0049)$.

For $\mathrm{EWI}_{4}$, the rates of patients with on-time drug pickup were extremely below the required target $(\geq 90 \%)$ during each of the three years. In details, the median performances varied from $36.5 \%$ [IQR: $28-47 \%$ ] in 2008 , to $15 \%$ [IQR: $10-21 \%$ ] in 2009 , and to $33 \%$ [IQR: $21-37 \%$ ] in 2010; with a statistically significant difference overtime $(\mathrm{p}=0.0132)$.

For $\mathrm{EWI}_{5}$, drug availability, also known as continuity in drug supply, decreased significantly $(\mathrm{p}=0.006)$ from $90 \%(9 / 10)$ in 2008 to $20 \%(2 / 10)$ in 2009 , then later reported a non-significant increment $(\mathrm{p}=0.625)$ to $40 \%$ $(4 / 10)$ in 2010. Interestingly, throughout the three years, the overall performance showed a significant drop down in drug supply overtime $(\mathrm{p}=0.023)$.

The overall trends (from 2008-2010) of the five HIVDR EWIs under study are reported in Figure 1, 


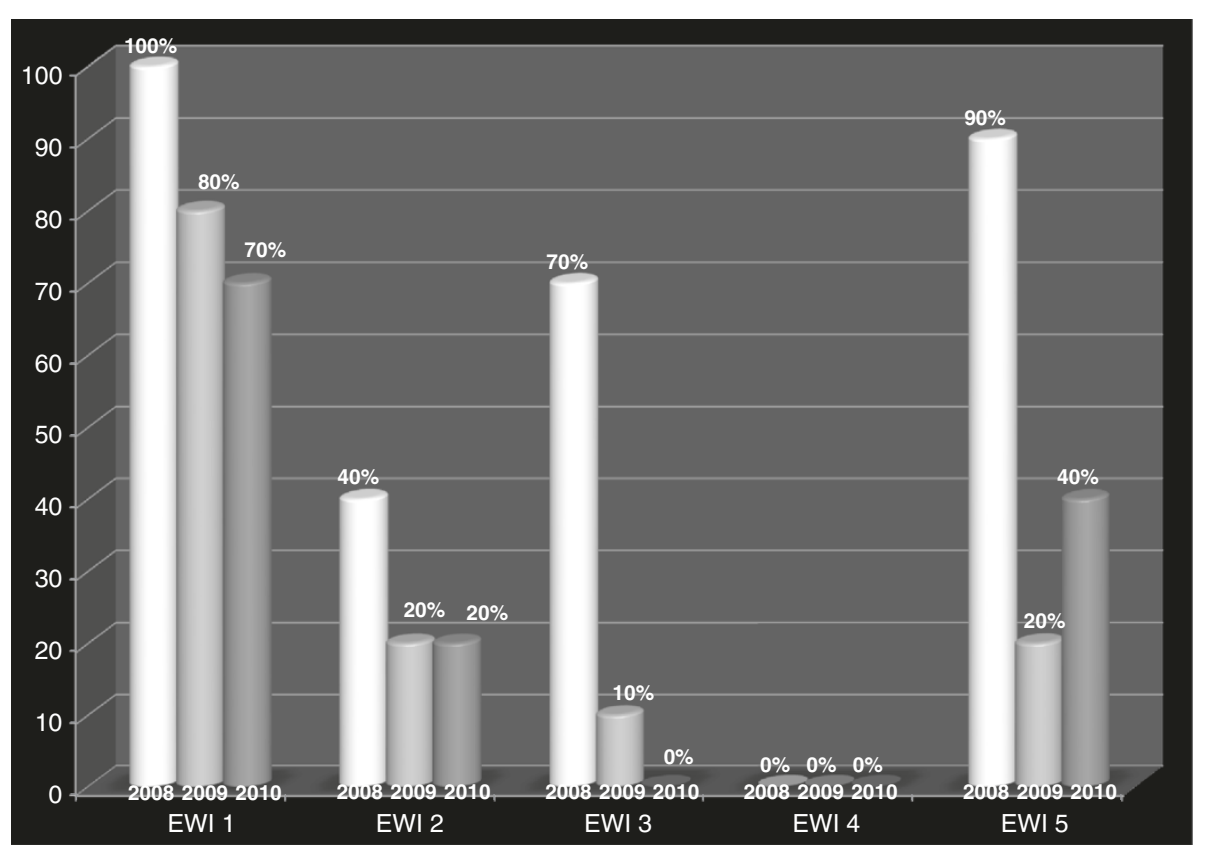

Figure 1 Trends of EWIs from 2008-2010. EWI : Early Warning Indicator 1, EWI2: Early Warning Indicator 2, EWl3: Early Warning Indicator 3, EWI4: Early Warning Indicator 4, EWI5: Early Warning Indicator 5. For each EWI, the graphs represent the national performance in 2008, 2009, and 2010, respectively.

showing the majority of clinics having optimal performances on prescribing practices $\left(\mathrm{EWI}_{1}\right)$ without any statistically significance, while the other four EWIs revealed suboptimal and decreasing performances overtime, most often (3/4) with significant differences overtime. The detail performance of EWIs, during each of the three years, and in each of the 10 surveyed ART clinics, is reported in Table 2.

\section{Discussions}

The increasing need to control HIVDR in low-resource settings (LRS) requires the implementation of efficient and rapid approaches to limit the emergence of preventable HIVDR, among which a population-based survey, such as EWI evaluations, could yield relevant evidences based-recommendations for best clinical practices. Overall, this is a paper on a topic of high importance in HIV medicine, transmitted drug resistance, as well as the methods for detecting it in a LRS where genotyping is not available, and remains challenging and clinically important issues to all the stakeholders who are involved in the global scale up of ART, particularly in Africa.

Physicians' prescribing practices $\left(\mathrm{EWI}_{1}\right)$ remained nationwide in conformity with the national guidelines. Interestingly, even sites with the $98-99 \%$ performances for $\mathrm{EWI}_{1}$ had no cases of dual- or mono-ARV prescriptions, which are more likely to select for drug resistant HIV rather than an inappropriate prescribing of drugs such as boosted protease inhibitors, abacavir, or tenofovir (which may not be appearing on several guidelines) which likely have less significance related to HIVDR. This good practice was mainly due to the use of standardized ART regimens and the institution of a consortium agreement (therapeutic committee) for every treatment initiation. This is a weekly consortium body made up of health staff all trained in HIV/AIDS management (medical doctors, nurses, medical biologists/laboratory technicians, medical counselors, pharmacists/pharmacy clerks, data manager and/or community relay agents; a practice which merits to be encouraged. Globally, $75 \%$ of clinics monitored worldwide met the WHO-recommended target on prescribing practices [16]. Specifically, other African countries (Malawi, Namibia, and others) also reported good practices [18-24], while poor performances were observed out of Africa (Central American, Caribbean, Asian, Oceanic and Western Pacific: from 38 to 75\%) [23-31]. Lack of evaluation overtime in the above mentioned studies could not enable in-depth comparison to ours. Still, the use of a therapeutic committee as well as continuous training may be vital practices to maintain and/or improve good ARV prescriptions.

The high and increasing rates of lost-to-follow-up are indicative of a growing rate of patients harboring potential drug resistant viruses within the national context, which in turns supports a growing risk of HIVDR emergence in Cameroon. These would have been favored by the observed heavy workload, and also probably due to termination of working contracts for community relay agents (CRA). Thus, task shifting (from medical doctors to nurses), and probably community empowerment (by 
Table 2 Site performances of EWI from 2008-2010

\begin{tabular}{|c|c|c|c|c|c|c|c|c|c|c|c|c|c|c|c|c|}
\hline \multirow[t]{2}{*}{$\begin{array}{l}\text { EWI per year } \\
\text { ART sites }\end{array}$} & \multicolumn{3}{|c|}{$\begin{array}{c}\text { EWI 1: Target }=100 \% \\
\text { (n/total) }\end{array}$} & \multicolumn{3}{|c|}{$\begin{array}{l}\text { EWI 2: Target } \leq 20 \% \\
\text { (n/total) }\end{array}$} & \multicolumn{3}{|c|}{$\begin{array}{l}\text { EWI 3: Target } \geq 70 \% \\
\text { (n/total) }\end{array}$} & \multicolumn{3}{|c|}{$\begin{array}{l}\text { EWI 4: Target } \geq 90 \% \\
\text { (n/total) }\end{array}$} & \multicolumn{3}{|c|}{$\begin{array}{l}\text { EWI 5: Target }=100 \% \\
\text { (n/total) }\end{array}$} & \multirow[t]{2}{*}{$\begin{array}{l}\text { Overall performance } \\
\text { per site } \% \text { (n/total) }\end{array}$} \\
\hline & 2008 & 2009 & 2010 & 2008 & 2009 & 2010 & 2008 & 2009 & 2010 & 2008 & 2009 & 2010 & 2008 & 2009 & 2010 & \\
\hline \multirow[t]{2}{*}{ Y.G.H } & $100 \%$ & $100 \%$ & $100 \%$ & $37 \%$ & $39 \%$ & $35 \%$ & $63 \%$ & $47 \%$ & $56 \%$ & $47 \%$ & $19 \%$ & $29 \%$ & $92 \%$ & $33 \%$ & $58 \%$ & $20 \%(3 / 15)$ \\
\hline & $(30 / 30)$ & $(200 / 200)$ & $(135 / 135)$ & $(11 / 30)$ & (77/198) & $(47 / 135)$ & $(19 / 30)$ & $(94 / 200)$ & $(76 / 135)$ & $(14 / 30)$ & (37/192) & (39/135) & $(11 / 12)$ & $(4 / 12)$ & $(7 / 12)$ & \\
\hline \multirow[t]{2}{*}{ H. CNPS } & $100 \%$ & $100 \%$ & $99 \%$ & $10 \%$ & $20 \%$ & $20 \%$ & $90 \%$ & $70 \%$ & $56 \%$ & $30 \%$ & $39 \%$ & $37 \%$ & $100 \%$ & $50 \%$ & $100 \%$ & $60 \%(9 / 15)$ \\
\hline & $(30 / 30)$ & $(200 / 200)$ & $(158 / 160)$ & $(3 / 30)$ & $(39 / 194)$ & $(20 / 132)$ & $(27 / 30)$ & $(133 / 193)$ & $(90 / 160)$ & $(9 / 30)$ & $(78 / 200)$ & $(58 / 157)$ & $(12 / 12)$ & $(6 / 12)$ & $(12 / 12)$ & \\
\hline \multirow[t]{2}{*}{ Y.C.H } & $100 \%$ & $100 \%$ & $100 \%$ & $27 \%$ & $31 \%$ & $39 \%$ & $77 \%$ & $38 \%$ & $55 \%$ & $28 \%$ & $12 \%$ & $32 \%$ & $100 \%$ & $92 \%$ & $67 \%$ & $33 \%(5 / 15)$ \\
\hline & $(30 / 30)$ & $(200 / 200)$ & $(180 / 180)$ & $(8 / 30)$ & $(62 / 200)$ & $(64 / 165)$ & $(23 / 30)$ & $(76 / 200)$ & $(94 / 170)$ & $(8 / 30)$ & $(24 / 200)$ & (58/180) & $(12 / 12)$ & $(11 / 12)$ & $(8 / 12)$ & \\
\hline \multirow[t]{2}{*}{ Y.J.H } & $100 \%$ & $100 \%$ & $100 \%$ & $10 \%$ & $46 \%$ & $43 \%$ & $77 \%$ & $27 \%$ & $50 \%$ & $3 \%$ & $15 \%$ & $56 \%$ & $100 \%$ & $75 \%$ & $75 \%$ & $40 \%(6 / 15)$ \\
\hline & $(30 / 30)$ & $(200 / 200)$ & $(155 / 155)$ & $(3 / 30)$ & $(86 / 186)$ & $(60 / 139)$ & $(23 / 30)$ & $(51 / 186)$ & $(73 / 145)$ & $(1 / 30)$ & $(29 / 196)$ & $(86 / 153)$ & $(12 / 12)$ & $(9 / 12)$ & $(9 / 12)$ & \\
\hline \multirow[t]{2}{*}{ D.G.H } & $100 \%$ & $100 \%$ & $99 \%$ & $40 \%$ & $41 \%$ & $38 \%$ & $60 \%$ & $47 \%$ & $36 \%$ & $50 \%$ & $57 \%$ & $50 \%$ & $100 \%$ & $25 \%$ & $100 \%$ & $27 \%(4 / 15)$ \\
\hline & $(30 / 30)$ & $(188 / 188)$ & $(158 / 160)$ & $(12 / 30)$ & $(76 / 185)$ & $(60 / 160)$ & $(18 / 30)$ & (89/188) & (58/159) & $(15 / 30)$ & $(113 / 199)$ & $(74 / 147)$ & $(12 / 12)$ & $(3 / 12)$ & $(12 / 12)$ & \\
\hline \multirow[t]{2}{*}{ L.H.D } & $100 \%$ & $100 \%$ & $99 \%$ & $37 \%$ & $29 \%$ & $33 \%$ & $63 \%$ & $66 \%$ & $60 \%$ & $43 \%$ & $21 \%$ & $34 \%$ & $100 \%$ & $100 \%$ & $100 \%$ & $33 \%(5 / 15)$ \\
\hline & $(30 / 30)$ & $(200 / 200)$ & $(158 / 160)$ & $(11 / 30)$ & $(58 / 200)$ & $(53 / 160)$ & $(19 / 30)$ & $(132 / 200)$ & $(93 / 156)$ & $(13 / 30)$ & $(41 / 194)$ & $(54 / 160)$ & $(12 / 12)$ & $(12 / 12)$ & $(12 / 12)$ & \\
\hline \multirow[t]{2}{*}{ N.D.H } & $100 \%$ & $100 \%$ & $100 \%$ & $33 \%$ & $15 \%$ & $58 \%$ & $70 \%$ & $35 \%$ & $44 \%$ & $30 \%$ & $10 \%$ & $12 \%$ & $100 \%$ & $50 \%$ & $100 \%$ & $47 \%(7 / 15)$ \\
\hline & $(30 / 30)$ & $(200 / 200)$ & $(175 / 175)$ & $(10 / 30)$ & $(25 / 162)$ & $(101 / 175)$ & $(21 / 30)$ & $(127 / 194)$ & $(70 / 159)$ & $(9 / 30)$ & $(19 / 200)$ & $(20 / 171)$ & $(12 / 12)$ & $(6 / 12)$ & $(12 / 12)$ & \\
\hline \multirow[t]{2}{*}{ L.R.H } & $100 \%$ & $99 \%$ & $100 \%$ & $27 \%$ & $40 \%$ & $19 \%$ & $73 \%$ & $57 \%$ & $44 \%$ & $13 \%$ & $2 \%$ & $0 \%$ & $100 \%$ & $100 \%$ & $42 \%$ & $40 \%(6 / 15)$ \\
\hline & $(30 / 30)$ & $(196 / 198)$ & $(160 / 160)$ & $(8 / 30)$ & $(80 / 200)$ & $(30 / 160)$ & $(22 / 30)$ & $(113 / 199)$ & $(68 / 153)$ & $(4 / 30)$ & (3/199) & $(0 / 160)$ & $(12 / 12)$ & $(12 / 12)$ & $(5 / 12)$ & \\
\hline \multirow[t]{2}{*}{ B.R.H } & $100 \%$ & $100 \%$ & $100 \%$ & $20 \%$ & $97 \%$ & $41 \%$ & $70 \%$ & $1 \%$ & $49 \%$ & $43 \%$ & $15 \%$ & $34 \%$ & $100 \%$ & $50 \%$ & $17 \%$ & $40 \%(6 / 15)$ \\
\hline & $(30 / 30)$ & $(200 / 200)$ & $(172 / 172)$ & $(6 / 30)$ & $(193 / 198)$ & $(68 / 167)$ & $(21 / 30)$ & $(2 / 200)$ & $(82 / 167)$ & $(13 / 30)$ & $(29 / 197)$ & $(50 / 146)$ & $(12 / 12)$ & $(6 / 12)$ & $(2 / 12)$ & \\
\hline \multirow[t]{2}{*}{ P.M.B } & $100 \%$ & $98 \%$ & $100 \%$ & $10 \%$ & $32 \%$ & $25 \%$ & $\mathbf{8 7} \%$ & $34 \%$ & $53 \%$ & $77 \%$ & $9 \%$ & $21 \%$ & $100 \%$ & $42 \%$ & $42 \%$ & $33 \%(5 / 15)$ \\
\hline & $(30 / 30)$ & $(176 / 180)$ & $(114 / 114)$ & $(3 / 30)$ & $(54 / 170)$ & $(28 / 114)$ & $(26 / 30)$ & $(60 / 180)$ & $(62 / 117)$ & $(23 / 30)$ & $(19 / 211)$ & $(26 / 125)$ & $(12 / 12)$ & $(5 / 12)$ & $(5 / 12)$ & \\
\hline
\end{tabular}


allocating resources to CRAs), as well as free consultations and reduced laboratory costs in such poor settings, may help in reducing the rate of missing patients. Indeed, the numbers of required health care workers to provide ART in LRS (1-2 physicians/1000 patients, 2-7 nurses/1000 patients, $<1-3$ pharmacy staff/1000 patients, and wider ranges for other health-allied staffs) estimated by Hirschhorn et al. [32], was based on the WHO-3by5 target (i.e. treatment of 5 million patients by 2005), and currently need to be revised in order to match the growing ART coverage (presently $>8$ millions) [1]. With restriction to the WHO-protocol, a direct evidence-based evaluation has not yet been conducted to investigate on the real impact of CRA's disengagement on lost to follow-up. Globally, 69\% of clinics monitored worldwide met the WHO-recommended target [16]. Particularly, in other African countries, lost-to-follow-up is also challenging (40 to 75\%) [18-24], whereas out of Africa higher performances were recorded (54 to 100\%) [23-31]. Furthermore, the impact of distance to the clinic, waiting time prior to medical consultation, stigma and patient educational level, are factors that may help in reducing lost patients [33]. Indeed, ART uptake has been negatively associated with distance from the nearest primary healthcare [34,35], thus indicating a possible need for creating new ART clinics to foster adherence.

The gradual poor retention of patients on first line ART after 12 months ART may be indicating a rapid switch to second line regimens that could be explained by numerous other factors (wider availability of HIV Viral Load testing, greater clinician experience/awareness on identifying treatment failure, a preexisting drug resistant mutation, etc.). Further studies are therefore needed to determine the time-to-treatment, to implement measures toward long term efficacy of first line ART and to limit events of inappropriate switch to second line ART in these settings. Furthermore, failure to be on first line therapy one year after initiation might in fact not necessarily be a negative indicator, since it might indicate a correct and necessary treatment switch to second line. This observation makes questionable the overall utility of $\mathrm{EWI}_{3}$, which would likely need to be revised for better utility in future. Globally, $67 \%$ of clinics monitored worldwide met the WHO-recommended target [16]. More interestingly, our performances in 2008 (70\%) were similar to those found in other African settings (among which Malawi: 53\% and Namibia: 67\%) [18-24], while higher performances (62-90\%) were recorded out of Africa [23-31]. Since first line ARV drugs $(\approx \$ 100 /$ patient/year) are about 4 times less costly than second line, an effective retention on first line is economical [36] and may regulate the current fast switch to second line ARV ( $2 \%$ of total patients on ART in 2006, to $13 \%$ of total patients on ART in 2009) in Cameroon [4]. These analyses are consistent with the different HIVDR rates to NRTI and NNRTI in 2003 (before ART scale-up: 5.6\% M184V and 6.1\%Y181C) and thereafter (following ART scale-up: $16.3 \% \mathrm{M} 184 \mathrm{~V}$ and $63.7 \% \mathrm{Y} 181 \mathrm{C}$ ) [37]. Despite the higher rate of transmitted HIVDR in Latin America (12-20\%), sub-Saharan Africa, though with only 4-9\% of transmitted HIVDR, is experiencing a rapid scale-up of ART associated with an increasing rate of transmitted HIVDR (38\% increasing risk of HIVDR per year): this important fact urgently needs a regular HIVDR surveillance system $[14,16,38]$.

The overall delay in drug pick-up strongly supports a national revision of the community engagement strategy to support patient adherence to ART programmes. This poor/ decreasing performance in drug pick-up $\left(\mathrm{EWI}_{4}\right)$, together with $\mathrm{EWI}_{2}$ and 3, clearly placed patient non-adherence as the main factor with high risks of HIVDR development and spread within the community. However, it should be noted that $\mathrm{EWI}_{4}$ itself seems problematic and may not be entirely practical to address adherence issues. Indeed, in our setting, clients may present one-to-two day(s) after their required drug pick-up appointment date and still maintain 100\% ART adherent, due to availability of remaining pills from the previous appointment. Thus, as for $\mathrm{EWI}_{3}$, the overall utility of $\mathrm{EWI}_{4}$ may also be brought into question, and would likely require expert review or reconsideration. Thus, the low scores found in our study are partly explained by the rigidity of this indicator, which has further being recently modified in the 2012 HIVDR report [16]. Globally, $70 \%$ of clinics monitored worldwide met the WHOrecommended target for timely drug keep-up [16]. Detail analysis also reports lower timely drug pick-ups in other African settings (between 17\% and 41\%) [18-24], against settings out of Africa (73-100\%) [23-31]. Thus, in African AIDS programmes, issues such as "time spent by patient for pharmacy service", "service quality rendered by pharmacy staff", "pharmacy localization", may be partly explained by delays in drug pick-up. As suggested by El-Khatib et al. in 2011, adherence to drug-refill (pill count) may also be a useful EWI of virologic and immunologic failure on first-line ART in African settings [23].

The gradual poor performances in drug supply present $E \mathrm{WI}_{5}$ as the main programmatic setback in the national ART performance. Despite the provision of alternative ART (by replacing shortage of efavirenz with nevirapine or lopinavir/ritonavir) in case of drug discontinuity, stock outs still negatively impact patient adherence with non-negligible risks of HIVDR due to potential suboptimal drug levels. Drug supply machinery should be urgently revised. Our findings could be strengthened by conducting further investigations to as to why these stock outs are occurring; targeting specifically the supply lines, trade agreements specific to Cameroon, land versus air versus sea route issues, or in-country distribution network 
problems. Globally, $65 \%$ of clinics monitored worldwide met the WHO-recommended target for drug supply. Geographically, the procurement systems were successful (100\%) in Malawi (cross-sectional study) [18], and with an increasing performance in the Caribbean (31.3\%-94\%, from 2007-2009) [23,24]. Aggregated data showed poorer performance in sub-Saharan Africa (42\%-47\%), as compared to non-African countries (32-90\%) [20-31]. Thus, African settings are more in need of further investigations to improve their drug supply system.

\section{Lessons learnt from the three year survey}

This study has effectively identified some strengths and weakness of the national ART programme, amongst which the encouraging prescribing practices, and corrective measures been addressed for other EWIs. Indeed, the performance of these later EWIs has decreased over time rather than staying steady or improving (as would have been hoped). Thus, this result is also very interesting, and if taken at face value, is a serious indictment of the overall implementation strategies underway in Cameroon, and a crucial cause for concern for generation of drug resistance. Therefore, tasks shifting/decentralization (to alleviate the heavy workload) and community re-empowerment are underway [4,39]. A specific algorithm (in form of posters and hand-outs) presenting overall performances and addressing adapted corrective actions, has been provided to guide healthcare providers working at the ART clinic (see Additional file 1). Also, advocacy, addressed to health authorities (ministry and heads of health facilities), boosted the implementation of the above corrective measures. Additionally, a bottom-to-top approach, through consideration of associations of people living with HIV, would allow patients to participate as key players in the success of the national ART performance [33,35,36,39]. Site supervision is also essential for an effective integration of HIVDR activities in the routine clinical practices, to make EWI an instrumental in prioritizing measures and allocating resources for clinics. Our analyses suggest that ART programmes in other LRS may be experiencing similar declining performances, and thus need relevant measures.

\section{Challenges resulting from our findings}

A successful and sustainable ART programme performance should be accompanied by scalable EWI survey in the country; the major setback relies on the regular availability of the required resources [12]. Secondly, despite the availability of external support, additional fund raising is still needed to optimize patient healthcare. Continuous staff training is essential to sustain good practices; brain drain makes the heavy workload persistent, thereby calling for policies to train and retain qualified personnel, especially with the need/creation of new ART clinics in the country to ensure scaling-up of the treatment programmes [36]. More importantly, recent studies in Cameroon showed low to moderate levels of transmitted HIVDR [40], and increasing levels of acquired HIVDR after 12 and 24 months [41], thus predicting growing risks of treatment failure and HIVDR to the commonly used drugs, due to a broad range of factors $[35,39]$. Without attempting to create a direct temporal relationship, the increasing rate of transmitted and acquired HIVDR in Cameroon, alongside the increasing/widely availability of ARV treatment, supports also a growing need of affordable viral load and HIVDR testing, and a more regular surveillance of HIVDR in this country [8,38]. Pediatric HIVDR surveillance is of prime importance, and needs to be implemented [42]. Of note, as observed in other countries, Fokam et al. also found low (4.9\%) and high (90\%) rates of HIVDR among drug-naïve and first-line ART failing children in Cameroon, respectively [43]. Finally, the emerging HIV co-infections with tuberculosis, malaria, viral hepatitis, require setting-up an antimicrobial drug resistance strategic plan and working group to preserve active drugs for the next generation $[44,45]$.

Despite restrictions to WHO-standards, our study limitations could not allow greater/meaningful statistical analysis, due to the limited number of sampled ART sites. Furthermore, difficulties in evaluating other factors that could potentially affect ART performances (disengagement of CRAs, task shifting, distance to clinic, stigma/discrimination, educational level, bottle nets in the drug supply system, etc.) also limited the strengths of our recommendations.

\section{Conclusions}

The extended use of ART in developing countries warrants a thorough understanding of all factors contributing to the success of national ART programmes. In the Cameroonian setting, patient adherence, drug stock outs, a potential community disengagement and a probable heavy workload, appear as major factors favoring an increasing risk of HIVDR emergence.

Use of EWI is an effective approach to identify both patient and programmatic factors favoring the risks of HIVDR at the clinics and at the national levels. With its simplicity, low cost, and rapid evidence-based policymaking, such surveys would be useful for a successful scale-up of ART in LRS, which in turns contribute to the global HIVDR prevention and surveillance strategy.

\section{Additional file}

Additional file 1: Recommendations to limit HIV Drug Resistance in Cameroon.

\section{Competing interests}

Authors declare that they have no financial, personal, or professional interests that could be construed to have influenced the paper. 


\section{Authors' contributions}

Conceived and designed the study: GDN, SCB, JF, ACZKB, EK, PM, IA, ASN, AFA, PMN, VC, JBNE. Acquired the data: ZT, SCB, JF, EK, PM, IA, ASN, GDN. Analyzed and interpreted the data: JF, SCB, EK, PM, ZT, GDN, IA, JBNE. Drafted the manuscript: JF, SCB, EK, PM, GDN, JBNE. Revised the manuscript: $A C Z B, I A, A S N, Z T, A F A, P M N, V C$. Approved the final version of the manuscript: SCB, JF, ACZB, EK, PM, IA, ASN, AFA, ZT, GDN, PMN, VC, JBNE. All authors read and approved the final manuscript.

\section{Acknowledgements}

This work was supported by ResNet, the World Health Organization (WHO) Global HIV Drug Resistance Surveillance network

We would like to thank Michael Jordan, Diane Bennett and Patrick Kombate-Noudjo, for providing their expertise in implementing our national HIV Drug Resistance strategic plan.

We also thank Charlie Alain Mbo'o, Isaac Macauley, Peter Tendoh, Arlette Messeh, of the national HIVDR working group for participating in the data collection. The manuscript was entirely reviewed by Odile Ouwe Missi Oukem Boyer of the Chantal BIYA International Reference Centre for research on HIV/AIDS prevention and management (CIRCB) in Yaounde, Cameroon.

\section{Author details}

${ }^{1}$ Chantal BIYA International Reference Centre (CIRCB) for research on HIV/ AIDS prevention and management, Yaounde, Cameroon. ${ }^{2}$ Faculty of Medicine and Biomedical Sciences (FMBS) of the University of Yaounde 1, Yaounde, Cameroon. ${ }^{3}$ Central Technical Group (CTG), National AIDS Control Committee (NACC), Yaounde, Cameroon. ${ }^{4}$ Department of Disease Control, Ministry of Public Health, Yaounde, Cameroon. ${ }^{5}$ World Health Organisation (WHO) Afro, National Office, Yaounde, Cameroon. ${ }^{6}$ Division of Operational Health Research (DROS), Ministry of Public Health, Yaounde, Cameroon. ${ }^{7}$ Approved Treatment Centre, Yaoundé Central Hospital, Yaounde, Cameroon. ${ }^{8}$ Virology Laboratory, Centre de Recherche en Maladies Emergentes et Ré-émergentes (CREMER)/IMPM/IRD, Yaounde, Cameroon. ${ }^{9}$ Approved Treatment Centre (ATC), Yaounde General Hospital, Yaounde, Cameroon. ${ }^{10}$ Faculty of Health Sciences (FHS), University of Buea, Buea, Cameroon. ${ }^{11}$ UNESCO Biotechnology Chair, Department of Biological Sciences, University of Rome "Tor Vergata", Rome, Italy.

\section{Received: 16 November 2012 Accepted: 3 April 2013}

Published: 8 April 2013

\section{References}

1. United Nations: Political declaration on HIV and AIDS: intensifying our efforts to eliminate HIV and AIDS. New York; 2011. http://www.unaids.org/en/ aboutunaids/unitednationsdeclarationsandgoals/ 2011 highlevelmeetingonaids, accessed 15 September 2012

2. WHO, UNAIDS and UNICEF: Global HIV/AIDS response: epidemic update and health sector progress towards universal access. Progress Report 2011. Geneva: World Health Organization; 2011. http://www.who.int/hiv/pub/ progress_report2011/en/index.html, accessed 15 September 2012.

3. Institut National de la Statistique (INS), République du Cameroun: Enquête Démographique de Santé (EDS IV). Yaounde: Rapport; 2011.

4. HIV Drug Resistance Working Group: Activités de Prévention et de Surveillance des Résistances du VIH aux Médicaments Antirétroviraux au Cameroun. Yaoundé: National AIDS Control Committee; Rapport Annuel; 2010.

5. WHO/UNAIDS: The WHO and UNAIDS global initiative to provide antiretroviral therapy to 3 million people with HIV/AIDS in developing countries by the end of 2005. Geneva: World Health Organization; 2003. Available at: http://www. who.int/3by5/publications/documents/en. Accessed 2008 Aug 05.

6. World Health Organization: Antiretroviral Therapy for HIV Infection in Adults and Adolescents in Resource-Limited Settings: Towards Universal Access: Recommendations for a public health approach. Geneva: World Health Organization; 2006. ISBN: 9789241599764 . Available at: http://www.who.int/ hiv/pub/arv/adult2010/en/index.html. Accessed 2010 Dec 10.

7. Gallant JE: Antiretroviral drug resistance and resistance testing. Top HIV Med 2005-2006, 13(5):138-142.

8. Fokam J, Salpini R, Santoro MM, Cento V, D'Arrigo R, Gori C, Perno CF, Colizzi V, Nanfack A, Gwom LC, Cappelli G, Takou D: Performance evaluation of an in-house human immunodeficiency virus type-1 protease-reverse transcriptase genotyping assay in Cameroon. Arch Virol 2011, 156(7):1235-1243.
9. Yang C, McNulty A, Diallo K, Zhang J, Titanji B, Kassim S, Wadonda-Kabondo N, Aberle-Grasse J, Kibuka T, Ndumbe PM, Vedapuri S, Zhou Z, Chilima B, Nkengasong JN: Development and Application of a Broadly Sensitive Dried-Blood-Spot-Based Genotyping Assay for Global Surveillance of HIV-1 Drug Resistance. J Clin Microbiol 2010, 48(9):3158-3164.

10. Bennett DE, Bertagnolio S, Sutherland D, Gilks CF: The World Health Organization's global strategy for prevention and assessment of HIV drug resistance. Antivir Ther 2008, 13(Suppl 2):1-13.

11. Jordan MR, Bennett DE, Bertagnolio S, Gilks CF, Sutherland D: World Health Organization surveys to monitor HIV drug resistance prevention and associated factors in sentinel antiretroviral treatment sites. Antivir The 2008, 13(Suppl 2):15-23.

12. HIV Drug Resistance Working Group: Guidelines for the evaluation of Early Warning Indicators for HIV drug resistance (EWI). Yaounde: National AIDS control committee; 2010

13. Bennett DE, Myatt M, Bertagnolio S, Sutherland D, Gilks CF: Recommendations for surveillance of transmitted HIV drug resistance in countries scaling up antiretroviral treatment. Antivir Ther 2008, 13(Suppl 2):25-36.

14. Bennett DE: The requirement for surveillance of HIV drug resistance within antiretroviral roll out in the developing world. Curr Opin Infect Dis 2006, 19(6):607-614.

15. World Health Organization indicators: HIV Drug resistance Early Warning Indicators. World Health Organization indicators to monitor HIV drug resistance prevention at antiretroviral treatment sites. Geneva: World Health Organization indicators; 2010. Available at: http://new.paho.org/hq/ dmdocuments/2010/hivdr-early-warning-indicators-updated-april-2010.pdf, accessed on 25 January 2013.

16. World Health Organisation (WHO): WHO HIV Drug Resistance Early Warning Indicator Meeting Report. 2012. http://apps.who.int/iris/bitstream/10665/ 75183/1/9789241503938_eng.pdf, accessed on 25 January 2013.

17. Jordan MR, Bennett DE, Wainberg MA, Havlir D, Hammer S, Yang C, Morris L, Peeters M, Wensing AM, Parkin N, Nachega JB, Phillips A, De Luca A, Geng E, Calmy A, Raizes E, Sandstrom P, Archibald CP, Perriëns J, McClure CM, Hong SY, McMahon JH, Dedes N, Sutherland D, Bertagnolio S: Update on World Health Organization HIV drug resistance prevention and assessment strategy: 2004-2011. Clin Infect Dis 2012 May, 54(Suppl 4):S245-S249.

18. Hedt BL, Wadonda-Kabondo N, Makombe S, Harries AD, Schouten EJ, Limbambala E, Hochgesang M, Aberle-Grosse J, Kamoto K: Early warning indicators for HIV drug resistance in Malawi. Antivir Ther 2008, 13(Suppl 2):69-75.

19. Hong SY, Jonas A, Dumeni E, Badi A, Badi A, Pereko D, Blom A, Muthiani VS, Shiningavamwe AN, Mukamba J, Andemichael G, Barbara R, Bennett DE, Jordan MR: Population-Based Monitoring of HIV Drug Resistance in Namibia with Early Warning Indicators. J Acquir Immune Defic Syndr 2010, 55(4):27-31.

20. Dzangare J, Gonese E, Mugurungi O, Shamu T, Apollo T, Bennett DE, Kelley KF, Jordan MR, Chakanyuka C, Cham F, Banda RM: Monitoring of early warning indicators for HIV drug resistance in antiretroviral therapy clinics in Zimbabwe. Clin Infect Dis 2012, 54(Suppl 4):S313-S316.

21. El-Khatib Z, Katzenstein D, Marrone G, Laher F, Mohapi L, Petzold M, Morris L, Ekström AM: Adherence to Drug-Refill Is a Useful Early Warning Indicator of Virologic and Immunologic Failure among HIV Patients on First-Line ART in South Africa. PLoS One 2011, 6(3):e17518.

22. Sigaloff KC, Hamers RL, Menke J, Labib M, Siwale M, Ive P, Botes ME, Kityo C, Mandaliya K, Wellington M, Osibogun A, Geskus RB, Stevens WS, van Vugt M: Rinke de Wit TF; PharmAccess African Studies to Evaluate Resistance: Early warning indicators for population-based monitoring of HIV drug resistance in 6 African countries. Clin Infect Dis 2012, 54(Suppl 4):S294-S299.

23. Bennett DE, Jordan MR, Bertagnolio S, Hong SY, Ravasi G, McMahon JH, Saadani A, Kelley KF: HIV drug resistance early warning indicators in cohorts of individuals starting antiretroviral therapy between 2004 and 2009: World Health Organization global report from 50 countries. Clin Infect Dis 2012, 54(Suppl 4):S280-S289.

24. Jordan M, Kelley K, Hassani SA, Obeng-Aduasare Y, Bertagnolio S: Monitoring ART: Clinic and Program Performance Using WHO HIV Drug Resistance Early Warning Indicators in 21 Countries. $18^{\text {th }}$ CROI. Boston: Paper \# 626; 2011. Feb 27 - March 02.

25. Ravasi G, Pérez-Rosales MD, Mejía CVM, Zepeda K, Pérez ME, Aguilar R, Ramos K, Sued O, Alonso Gonzalez M, Israel K: WHO HIV drug resistance 
early warning indicators as treatment program monitoring and evaluation tool in Central American countries. IAS 2011.

Rome: TUAD 0105; 2011.

26. Noreen J, Ravasi G, Schrooten W, Sutherland D, Edwards P, Del Riego A: HIV Drug Resistance Early Warning Indicators in the Caribbean. In Caribbean HIV Conference. The Bahamas: Bahamas; 2011.

27. Ma Y, Zhang F, Li H, Wu H, Zhang J, Ding Y, Zhao D, Fang H, Zhou S, Liu Z, Zhang L, Osborne C, Seguy N, Zhao Y: Monitoring HIV drug resistance using early warning indicators in China: results from a pilot survey conducted in 2008. Clin Infect Dis 2012, 54(Suppl 4):S300-S302.

28. Daoni E, Kitur U, Parunga A, Ndenzako F, Lloyd A, Yu D: Experience in piloting HIV drug resistance early warning indicators to improve the antiretroviral program in Papua New Guinea. Clin Infect Dis 2012, 54(Suppl 4):S303-S305.

29. Do TN, Nguyen TM, Do MH, Masaya K, Dang TB, Pham TL, Yoshikawa K, Cao TT, Nguyen TT, Bui DD, Nguyen VK, Nguyen TL, Fujita M: Combining cohort analysis and monitoring of HIV early-warning indicators of drug resistance to assess antiretroviral therapy services in Vietnam. Clin Infect Dis 2012, 54(Suppl 4):S306-S312.

30. Jack N, Ravasi G, Schrooten W, Sutherland D, Ghidinelli M, Del Riego A: Implementing early-warning indicators of HIV drug resistance in the Caribbean. Clin Infect Dis 2012, 54(Suppl 4):S290-S293.

31. Ravasi G, Jack N, Alonso Gonzalez M, Sued O, Pérez-Rosales MD, Gomez B, Vila M, Riego A, Ghidinelli M: Progress of implementation of the World Health Organization strategy for HIV drug resistance control in Latin America and the Caribbean. Rev Panam Salud Publica 2011, 30(6):657-662.

32. Hirschhorn LR, Oguda L, Fullem A, Dreesch N, Wilson P: Estimating health workforce needs for antiretroviral therapy in resource-limited settings. Hum Resour Health 2006, 4:1. doi:10.1186/1478-4491-4-1.

33. Atanga NP, Bin KL, Malfertheiner P, Christoph JA: My Friend with HIV Remains a Friend": A Novel HIVIAIDS Stigma Reduction Project in Cameroon. Addis Ababa: ICASA; 2011. WEPDD 0303.

34. Monge S, Jarrin I, Perez-Hoyos S, Ferreros I, García-Olalla P, Muga R, Del Romero J, Belda J, Castilla J, Bolúmar F, Del Amo J, GEMES: Educational level and HIV disease progression before and after the introduction of HAART. A cohort study in 989 HIV seroconverters in Spain. Sex Transm Infect 2011, 87(7):571-576.

35. Cooke GS, Tanser FC, Bärnighausen TW, Newell ML: Population uptake of antiretroviral treatment through primary care in rural South Africa. BMC Public Health 2010, 10:585.

36. Comité Nationale de Lutte contre le SIDA au Cameroun: Accès universel pour la PTME, la prise en charge globale des PVIH et la prévention du VIH auprès des populations les plus exposées au Cameroun. Proposition VIH du Cameroun--Série 10 (Global Fund Round 10).

37. Torimiro JN, Takou D, Salpini R, Nanfack A, Fokam J, Cappelli G, Perno CF, Colizzi V: Population Level Drug Resistance Mutations in HIV Type 1 Protease and Reverse Transcriptase in Cameroon: 1995 to 2010 Review. J Acquir Immune Defic Syndr 2011, 56:83.

38. Aghokeng AF, Kouanfack C, Laurent C, Ebong E, Atem-Tambe A, Butel C, Montavon C, Mpoudi-Ngole E, Delaporte E, Peeters M: Scale-up of antiretroviral treatment in sub-Saharan Africa is accompanied by increasing HIV-1 drug resistance mutations in drug-naive patients. AIDS 2011, 25(17):2183-2188.

39. Boyer S, Eboko F, Camara M, Abé C, Nguini ME, Koulla-Shiro S, Moatti JP: Scaling up access to antiretroviral treatment for HIV infection: the impact of decentralization of healthcare delivery in Cameroon. AIDS 2010, 24(Suppl 1):S5-S15.

40. Aghokeng FA, Vergne L, Mpoudi NE, Mbangue M, Deoudje N, Mokondji E, Nambei WS, Peyou-Ndi MM, Moka JJ, Delaporte E, Peeters M: Evaluation of transmitted HIV drug resistance among recently-infected antenatal clinic attendees in four Central African countries. Antivir Ther 2009, 14(3):401-411.

41. Kouanfack C, Montavon C, Laurent C, Aghokeng A, Kenfack A, Bourgeois A, Koulla-Shiro S, Mpoudi-Ngole E, Peeters M, Delaporte E: Low levels of antiretroviral-resistant HIV infection in a routine clinic in Cameroon that uses the World Health Organization (WHO) public health approach to monitor antiretroviral treatment and adequacy with the WHO recommendation for second-line treatment. Clin Infect Dis 2009, 48(9):1318-1322.

42. World Health Organization: Antiretroviral Therapy for HIV infection in children and infants: Toward Universal Access. Recommendations for public health approach. World Health Organization 2010 revision. Geneva: World Health Organization; 2010:83-86. Available at: www.who.int/hiv/pub/guidelines/art/ en. Accessed 2011 Apr 10.

43. Fokam J, Salpini R, Santoro MM, Cento V, Perno CF, Colizzi V, Ndumbe PM Fokunang Ntungen C, Ndiang Tetang SM, Nanfack AJ, Takou Komego DA, Cappelli G: Drug Resistance Among Drug-naive and First-line Antiretroviral Treatment-failing Children in Cameroon. Pediatr Infect Dis J 2011, 30(12):1062-1068.

44. Hedt BL, Laufer MK, Cohen T: Drug resistance surveillance in resource-poor settings: current methods and considerations for TB, HIV, and malaria. Am J Trop Med Hyg 2011, 84(2):192-199.

45. Beith A: Mapping Factors that Drive Drug Resistance (with a Focus on Resource-Limited Settings): A First Step Towards Better Informed Policy. Drug Resistance Working Group Background Paper. Washington DC: Center for Global Development; 2008:71. Available at: http://www.cgdev.org/doc/drug \%20resistance/web/DRWG_Characterization_Paper_16\%20July. Accessed 2011 Aug 18.

doi:10.1186/1471-2458-13-308

Cite this article as: Fokam et al:: Declining trends in early warning indicators for HIV drug resistance in Cameroon from 2008-2010: lessons and challenges for low-resource settings. BMC Public Health 2013 13:308.

\section{Submit your next manuscript to BioMed Central and take full advantage of:}

- Convenient online submission

- Thorough peer review

- No space constraints or color figure charges

- Immediate publication on acceptance

- Inclusion in PubMed, CAS, Scopus and Google Scholar

- Research which is freely available for redistribution

Submit your manuscript at www.biomedcentral.com/submit
C Biomed Central 